\title{
Aberration-Corrected STEM Investigation of Metallic Ti Cluster Formation in Mg-Ti Hydrides
}

D.M. Kepaptsoglou*, I.J.T. Jensen*, M. Sarahan**, Q.M. Ramasse**, H. Schreuders***, B. Dam***, S. Diplas****, O. M. Løvvik*,**** and J. Taftø*

*Department of Physics, University of Oslo, P.O. Box 1048, Blindern, 0316 Oslo, Norway

${ }^{* *}$ SuperSTEM Laboratory, STFC Daresbury, Keckwick Lane, Warrington, WA4 4AD, UK

${ }^{* * *}$ Materials for Energy Conversion or Storage (MECS), DelftChemTech, Faculty of Applied Science, Technical University Delft, P/O Box 5045, NL-2600 GA Delft, The Netherlands

${ }^{* * * *}$ SINTEF Materials and Chemistry, P.O. Box 124, Blindern, 0314 Oslo, Norway

$\mathrm{Mg}$ hydrides represent a very interesting category of materials due to their high hydrogen storage capacity as well as their strong potential for optical and electric properties and applications that extend from electronic devices to solar cells. Crucially, the addition of Ti to the Mg hydride can lead to significant improvement of its hydrogenation properties [1]. Although Ti exhibits a very low solubility in $\mathrm{Mg}$ and the non-equilibrium intermetallic phases of the two are highly metastable, accounts of single phase structure before and after hydrogenation have been reported in the literature [2]. Experimental evidence [3] and density functional theory calculations [4] support the idea that short range order of $\mathrm{Ti}$ atoms within the MgTi inter-metallic, rather than random or quasi-random distribution of $\mathrm{Ti}$ atoms in a the $\mathrm{Mg}$ matrix is responsible for the increase of the solubility by of the two elements by drastically lowering their mixing enthalpy [4]. Ab-initio density of state (DOS) calculations in particular suggest that Ti forms an atomic environment resembling pure Ti [4].

In this work we present experimental evidence of the presence of Ti nanoclusters in the MgTi system by means of aberration corrected scanning transmission electron microscopy (STEM) and electron loss spectroscopy (EELS). All analytical work was carried out on an aberration-corrected VGHB501 dedicated STEM at the U.K. SuperSTEM facility. We studied and compared the microstructure and electronic configuration of 'unloaded' (hydrogen-free) and H-loaded $\mathrm{Mg}_{80} \mathrm{Ti}_{20}$ thin films deposited on single crystal Si wafers by RF magnetron sputtering. Multivariate statistical analysis [5] of 2-dimensional spectrum images of the loaded hydride film reveals localised changes in the fine structure of both the $\mathrm{Ti} \mathrm{L}_{2,3}$ edge as well as the low loss valence spectra region. Customdeveloped nonnegative matrix factorization (NMF) methods were used to separate the resulting spectral components into metallic and oxidised $\mathrm{Ti}^{4+}$ and $\mathrm{Ti}^{3+}$ contributions [6].

\section{References}

[1] R. Niessen and P. Notten, Electrochem . Solid-State Lett, 8 (2005) A534

[2] D.M. Borsa et al, Phys. Rev B 75 (2007)205408

[3] R. Gremaud et al, Phys Rec B 77 (2008) 144204

[4] I.J.T. Jensen et al, Phys Rev B 82 (2010) 174121

[5] M. Watanabe et al., Microscopy and Microanalysis 13, 1264 (2007). The MSA plugin for Digital Micrograph is available commercially from HREM Research Inc., www.hremresearch.com

[6] M.Sarahan et al., in preparation (2011) 

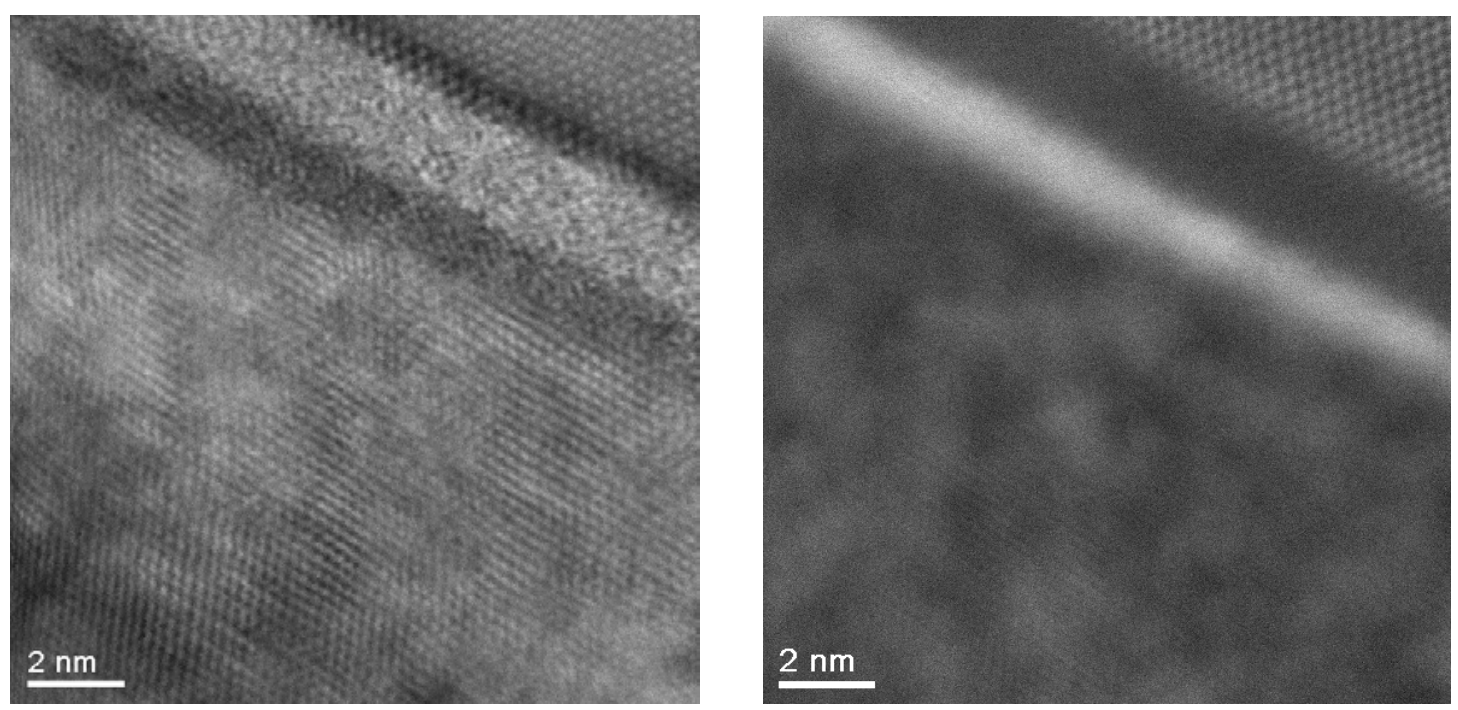

Figure 1: Bright (left) and HAADF (right) STEM images of the H-loaded $\mathrm{Mg}_{80} \mathrm{Ti}_{20}$ film deposited on a $\mathrm{Si}(100)$ wafer, showing 2 interfacial layers
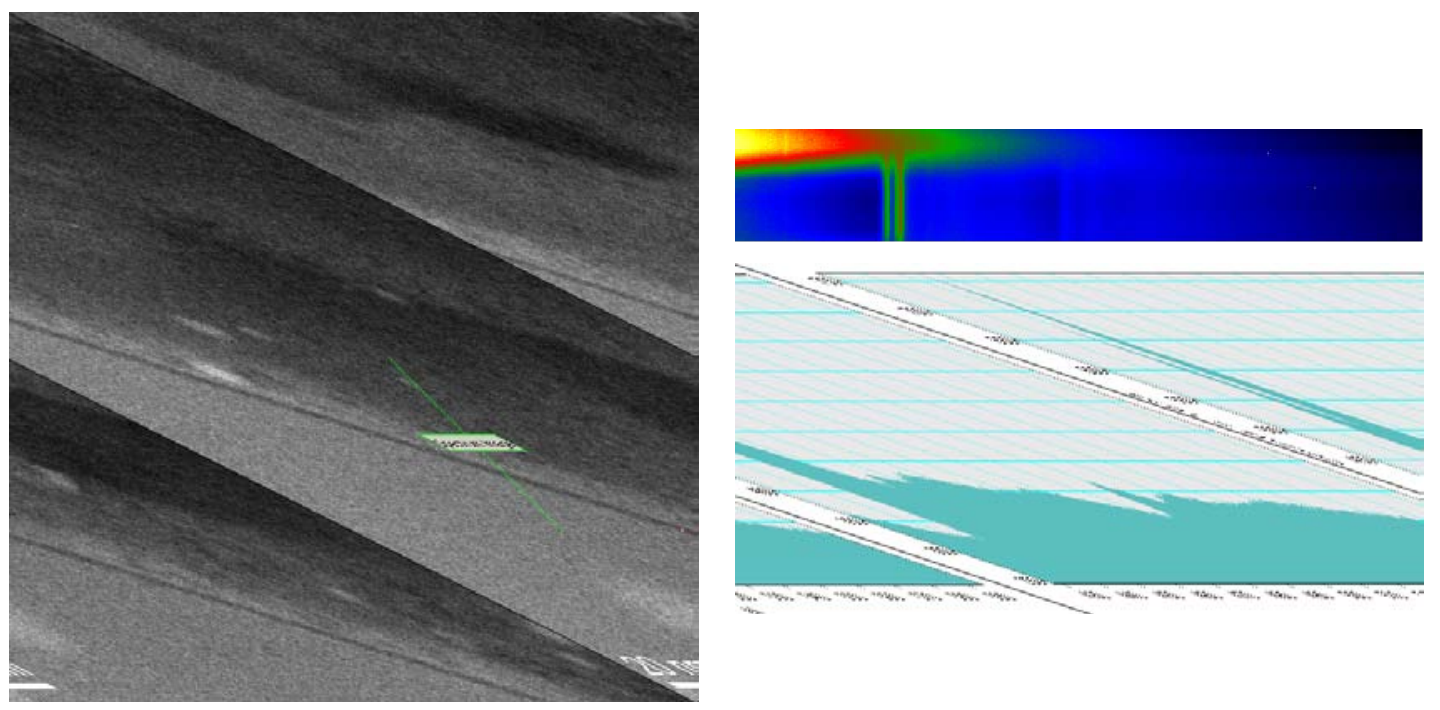

Figure 2: HAADF (left) STEM image of the H-loaded $\mathrm{Mg}_{80} \mathrm{Ti}_{20}$ film, Ti-L $\mathrm{L}_{2,3}$ ELNES spectrum image (top right) and ELNES spectrum exported from the SI (bottom right) 